\title{
Amplification of Modulated Waves in Magnetoactive Semiconductor Plasmas
}

\author{
Anita Sangwan ${ }^{1 *}$ \\ ${ }^{2}$ Department of Physics \\ Singhania University, Pacheri Bari \\ Jhunjunu-333515 (Rajasthan) India
}

\author{
Navneet Singh ${ }^{2}$ \\ ${ }^{2}$ Department of Physics \\ Rajiv Gandhi Govt. College for Women \\ Bhiwani-127021 (Haryana) India
}

\begin{abstract}
Using the hydrodynamic model of semiconductor plasmas and considering the origin of plasmon-longitudinal optical phonon interaction in effective third-order optical susceptibility, an analytical investigation of amplification of modulated waves is performed in magnetoactive semiconductor plasmas. Expressions for necessary threshold pump amplitude for the onset of amplification of modulated waves and growth rate of modulated wave have been obtained. Numerical estimations have been made for $n$-InSb crystal at $77 \mathrm{~K}$ illuminated by a $10.6 \mu \mathrm{m} \mathrm{CO}_{2}$ laser. The dependence of threshold pump amplitude and growth rate of modulated wave on wave number, externally applied magnetic field (via electron-cyclotron frequency) and doping concentration (via electron-plasma frequency) have been explored. It has been found that the threshold pump amplitude can be lowered whereas the growth rate of modulated wave can be enhanced by proper selection of externally applied magnetic field and doping concentration of semiconductor medium. The results of the present analytical investigation strongly manifest the importance of magnetoactive semiconductor plasmas as appropriate hosts for modulational instability processes.
\end{abstract}

Keywords-Modulational amplification, Fröhlich interaction, threshold pump amplitude, growth rate, semiconductor plasmas.

\section{INTRODUCTION}

Laser-semiconductor plasma interactions have been playing a prominent role in diverse areas of scientific research for several decades due to its immense applications in processing of materials and fabrication of devices [1,2]. Semiconductor plasmas also provide a compact and less expansive medium to model nonlinear optical phenomena encountered in laser technology. There exist a number of nonlinear interactions; the modulational interaction of coupled modes is significant one. In modulational interaction a strong space charge field modulates the pump wave [3]. Periodic variations of the propagation parameter lead to the modulation of an electromagnetic wave passing through the medium. The optical waves present in an electro-optic modulator can be strongly amplified through nonlinear optical pumping. The resulting amplification of decay channels by modulational interactions are generally referred to as an instability of wave propagating in nonlinear dispersive medium such that the steady-state becomes unstable and evolves into a temporally modulated state [4].

Modulational instabilities have played a very prominent role in diverse areas of scientific research. The devices based on modulational instability in semiconductor plasmas occupy a special place in nonlinear optical technology due to their prospective applications in fast optical communication and optoelectronic devices. This is due to the fact that semiconductor crystals are substantially transparent for photon energies much less than the band-gap energies and undergo optical damage at considerably large excitation intensities. In addition, semiconductors have added advantages over other materials in terms of compactness, control over the material relaxation times, observed large nonlinearities in optical properties under near resonant laser irradiation and sophisticated fabrication technology [5].

In semiconductor plasmas, the pump electric field interacts with both electrons as well as optical phonons (OPs). As a result of this interaction, the scattered radiation consists of two parts:

1. Single particle scattering, which is caused by individually moving electrons and is nearly elastic. This part of the spectrum can be used to determine the electron velocity distribution, and represents a great interest in transport theory.

2. Collective mode of scattering, which is caused by electron-plasma wave, i.e. plasmon (PL) mode in the electron gas.

In weakly-polar semiconductor plasmas, the collective modes are strongly coupled with the longitudinal optical phonon (LOP) mode via Fröhlich interaction, and one observes a frequency shift with increasing free carrier concentration [6]. The coupling of LOPs and free carrier collective excitations by macroscopic longitudinal electric fields in a polar semiconductor has been treated theoretically by Kaplan et.al. [7]. This coupled wave approach can also be generalized to include waves other than electromagnetic. However, in centrosymmetric crystals, one may replace the idler electromagnetic wave by an optically excited coherent collective mode such as the acoustical phonon mode, OP mode, polaron mode and polariton mode etc. A polaron is a quasiparticle that arises due to the conduction electron (or hole) together with its self-induced polarization in an ionic crystal or in a polar semiconductor $[8,9]$.

Here, it should be worth pointing out that Fröhlich interaction remains absent in covalent materials such as $\mathrm{Ge}$ and $\mathrm{Si}$, but it significantly affect the mobility of weakly-polar III-V semiconductors. For this type of studies III-V semiconductors happens to be obvious choice because of the possibility of rendering them p-type or n-type conductors through doping. At low doping levels, the interaction remains unscreened. With increasing doping levels, PLs and LOPs will no longer remain 
decoupled, rather the system will exhibit oscillations at coupled PL-LOP modes with frequency

$$
\omega_{ \pm}^{2}=0.5\left[\left(\omega_{p}^{2}+\omega_{\text {lop }}^{2}\right) \pm\left\{\left(\omega_{p}^{2}+\omega_{\text {lop }}^{2}\right)^{2}-4 \omega_{p}^{2} \omega_{\text {top }}^{2}\right\}^{1 / 2}\right],
$$

where $\omega_{p}=\left(n_{0} e^{2} / m \varepsilon\right)^{1 / 2}$ is the electron-plasma frequency and $\varepsilon=\varepsilon_{0} \varepsilon_{\infty}$ is the dielectric constant in which, $\varepsilon_{\infty}$ is the high frequency dielectric constant of the medium and $\varepsilon_{0}$ is the permittivity of free space. $\omega_{\text {lop }}$ and $\omega_{\text {top }}$ represent the LOP mode frequency and transverse optical phonon (TOP) frequency, respectively. $m$ is the effective mass of an electron of charge $-e$.

Hence, it would be interesting to find out the doping levels favorable for PL-LOP coupling in a degenerate polar semiconductor plasma. Since III-V semiconductor plasmas have been regarded as universally recognized nonlinear optical media with high optical nonlinearities which can be easily controlled by the application of external fields [10-12]. In the presence of externally applied magnetic field, the collective cyclotron excitations - LOPs coupling, via the macroscopic longitudinal electric field give rise to modified normal modes (i.e. polaron mode).

Under Voigt geometry, polaron mode frequency at the center of Brillouin zone (zero wave vector mode) is given by [13]

$$
\omega_{0, p o l}^{2}=0.5\left[\left(\omega_{p}^{2}+\omega_{c}^{2}+\omega_{l o p}^{2}\right) \pm\left\{\left(\omega_{p}^{2}+\omega_{c}^{2}+\omega_{l o p}^{2}\right)^{2}-4\left(\omega_{p}^{2} \omega_{\text {top }}^{2}-\omega_{c}^{2} \omega_{l o p}^{2}\right\}^{1 / 2}\right]\right.
$$

where $\omega_{c}=(\mathrm{e} / \mathrm{m}) B_{0}$ represent electron-cyclotron frequency in the presence of magnetostatic field $B_{0}$. It is clear that presence of magneto-plasma excitations modifies the coupling of PL-LOP quiet distinctively.

Literature survey reveals that up to now, a lot of theoretical work on the amplification of modulated waves induced by coherent collective modes in III-V semiconductor plasmas has been reported by several research groups [14-20], but PL-LOP interaction based amplification of modulated waves in weaklypolar magnetoactive semiconductor plasmas has yet to be studied. Thus, the aim of the present paper is just to investigate the amplification of modulated waves in magnetoactive semiconductor plasmas in the framework of a hydrodynamic model and coupled mode theory.

We choose the weakly-polar III-V semiconductor crystal, viz. $\mathrm{n}$-InSb as a model substance to have a polar OP scattering mechanism, so that coherent polaron mode may be excited through ultra fast excitation. An analytical investigation of the modulational interaction between the co-propagating pump beam and the internally generated polaron mode (due to the coupling of PLs and LOPs) is presented in a magnetoactive semiconductor medium: as a result of which it is found that the polaron mode is amplified at the expense of the pump wave. Further, by using coupled mode approach, strong tunable electromagnetic side bands may be achieved as a signal waves at the expense of the pump wave.

Nonetheless, the present analytical investigation has been made under the following assumptions:

1. All waves are infinite plane waves.

2. Interaction is phase matched.
3. Depletion of energy from the pump wave is being neglected.

4. Semiconductor plasma is assumed to be homogeneous, possessing isotropic non-degenerate parabolic band structure at moderately low temperature $(77 \mathrm{~K})$.

5. Pump photon energy is taken well below the band-gap energy of the sample (it allows the optical properties of the sample to be influenced considerably by free carriers and keeps it unaffected by the photo-induced inter-band transition mechanisms).

Finally Numerical estimations have been made with a set of data appropriate for a polar semiconductor plasma (n-InSb) duly irradiated by a frequency doubled $\mathrm{CO}_{2}$ laser to establish the validity of the present work.

\section{THEORETICAL FORMULATIONS}

In order to study the PL-LOP interaction based amplification of modulated waves in a magnetoactive semiconductor plasmas, the hydrodynamic model of homogeneous semiconductor plasma of infinite extent is considered. This model proves to be suitable for the present study as it simplifies our analysis, without any loss of significant information, by replacing the streaming electrons with an electron fluid described by a few macroscopic parameters like average carrier density, average velocity etc. However, it restricts our analysis to be valid only in the limit $k l$ $\ll 1$ ( $k$ is the wave number and $l$ is the carrier mean free path).

We consider the propagation of a spatially uniform pump wave $\vec{E}_{0}=\hat{x} E_{0} \exp \left(-i \omega_{0} t\right)$ in a weakly-polar semiconductor plasma medium subjected to a static magnetic field $\vec{B}_{0}=\hat{z} B_{0}$. Here, the incident pump wave is assumed spatially uniform $\left(\left|\vec{k}_{0}\right| \approx 0\right)$ because under dipole approximation the excited waves have wavelengths very small in comparison to the scalelength of the pump field variation [21] (i.e. $\left|\vec{k}_{0}\right| \ll|\vec{k}|$ so that $\vec{k}_{0}$ may be neglected).

The physics of the problem can be explained as follows: In the presence of two mutually perpendicular fields $\left(\vec{E}_{0}, \vec{B}_{0}\right)$, the motion of each free electron generates a macroscopic depolarization field normal to the surface of the crystal. The induced depolarization field strongly couples the longitudinal and transverse degrees of freedom of the medium and shifts the natural frequency away from the cyclotron frequency, and hence induces the collective cyclotron excitation with the resonance frequency $\omega_{c c}=\left(\omega_{p}^{2}+\omega_{c}^{2}\right)^{1 / 2}$. In the presence of pump wave, this collective cyclotron excitation generates a polaron mode via Fröhlich interaction (between PL and LOP modes). This interaction induces a very strong space charge field and derives the polaron mode at modulated frequency. Thus the pump wave and polaron mode in the medium can be strongly amplified through nonlinear optical pumping.

The basic equations employed in the present analysis are:

$$
\frac{\partial^{2} \vec{r}}{\partial t^{2}}+\left(\omega_{p}^{2}+\Gamma_{e}^{2}\right) \vec{r}+2 \Gamma_{e} \frac{\partial \vec{r}}{\partial t}=-\frac{e}{m}\left(\vec{E}_{0}+\frac{\partial \vec{r}}{\partial t} \times \vec{B}_{0}\right),
$$




$$
\frac{\partial^{2} \vec{s}}{\partial t^{2}}+\left(\omega_{l o p}^{2}+\Gamma_{p h}^{2}\right) \vec{s}+2 \Gamma_{p h} \frac{\partial \vec{s}}{\partial t}=\frac{q_{s}}{M}\left(\vec{E}_{0}+\frac{\partial \vec{s}}{\partial t} \times \vec{B}_{0}\right)
$$

Eqs. (3) and (4) are the linearised zeroth-order momentum transfer equations of the oscillatory electron fluid and lattice ions, respectively. $\Gamma_{e}$ and $\Gamma_{p h}$ represent the electron-electron collision frequency and the OP decay constant, respectively. These loss parameters have been introduced phenomenologically and do not vary with the pump and the externally applied magnetic fields. $s\left(=u^{+}+u^{-}\right)$is the relative displacement of positive and negative ions. These equations are similar to the PL-OP coupled mode equations [22].

The induced collective cyclotron vibration produces a polarization $P_{e}(=-n e r)$, the induced variations also influence the oppositely charged ions of a di-atomic crystal for the oscillations, and finally the oscillations result in an induced polarization $P_{s}\left(=N q_{s} s\right)$ of the medium. The effective charge of the lattice polarization is given by

$$
q_{s}=\omega_{l o p}\left[\frac{M}{N} \varepsilon_{0}\left(\frac{1}{\varepsilon}-\frac{1}{\varepsilon_{s}}\right)\right]^{1 / 2}
$$

where $M$ is the reduced mass of di-atomic molecule and $N$ $\left(=a^{3}\right)$ is the number of unit cells per unit volume; $a$ being the lattice constant of the crystal. We assume that the electric fields associated with the electronic and lattice polarizations are parallel to each other and the polarizabilities of the electrons and ion systems are additive. Thus, the simultaneous excitation of collective cyclotron vibrations and OPs result into coupling between them. The resulting coupled vibrations appear in the form of a new mode known as polaron mode. The equation of motion of a polaron mode is given by

$$
\frac{\partial^{2} \vec{R}}{\partial t^{2}}+\omega_{0, p o l}^{2} \vec{R}+2 \Gamma_{p o l} \frac{\partial \vec{R}}{\partial t}=(N M)^{1 / 2}\left(-\frac{e}{m}+\frac{q_{s}}{M}\right)\left[\vec{E}_{p o l}+\frac{\partial \vec{W}}{\partial t} \times \vec{B}_{0}\right]
$$

where $\vec{W}=\vec{r}+\vec{s}, \Gamma_{p o l}=\Gamma_{e}+\Gamma_{p h}$ (polaron mode decay constant) and $n=n_{0}+n_{1} \exp [i(k r-\omega t)] . E_{p o l}$ is the effective electrostatic field associated with polaron mode. Let us consider that the motion of polaron mode takes place with respect to a new origin shifted by some distance from the original position. Under this assumption, we may express the displacement of polaron mode in terms of new parameter $\vec{R}$ defined as $\vec{R}=(N M)^{1 / 2} \vec{W}$ [23].

Other basic equations governing the said modulational interaction are:

$$
\begin{aligned}
& \frac{\partial n_{1}}{\partial t}+n_{0} \frac{\partial v_{1}}{\partial x}+n_{1} \frac{\partial v_{0}}{\partial x}+v_{1} \frac{\partial n_{1}}{\partial x}=0, \\
& \frac{\partial E_{p o l}}{\partial x}+\left(\frac{N q_{s}}{\varepsilon_{0}}-\frac{n_{0} e}{\varepsilon_{0}}\right) \frac{\partial R}{\partial x}=\frac{-n_{1} e}{\varepsilon_{0}} .
\end{aligned}
$$

Eq. (7) is the continuity equation, in which $n_{0}$ and $n_{1}$ are the equilibrium and perturbed electron densities, respectively. $v_{0}$ and $v_{1}$ are the oscillatory fluid velocities of the electrons. Eq. (8) is the Poisson's equation, which determines the effective electrostatic field $E_{p o l}$ associated with polaron mode arising due to the induced electronic and lattice polarizations. We also assume that the energy transfer between the pump wave, polaron mode and side band modes satisfy phase matching conditions, which are:

$$
\hbar \vec{k}_{ \pm}=\hbar \vec{k}_{0} \pm \hbar \vec{k}_{p o l} \text { and } \hbar \omega_{ \pm}=\hbar \omega_{0} \pm \hbar \omega_{p o l},
$$

known as momentum and energy conservation relations, respectively. Under spatially uniform laser irradiations $\left|\vec{k}_{0}\right| \approx 0$, so that $\left|\vec{k}_{ \pm}\right| \approx\left|\vec{k}_{0} \pm \vec{k}_{p o l}\right| \approx\left|\vec{k}_{p o l}\right|=k$ (say).

Physically the polaron is an electron in a localized state that induces a polarization of the medium. This polarization is local in character and is due to the displacements of ions from the equilibrium positions caused by the field produced by the electron density, which gives rise to an electron density perturbation at the polaron mode frequency, which couples nonlinearity with the pump wave and derives the polaron mode at the modulated frequency. Following the procedure adopted by Singh et.al. [24] and using Eqs. (3) and (6), we obtain

$$
\begin{array}{r}
\frac{\partial^{2} n_{1}\left(\omega_{ \pm}, k_{ \pm}\right)}{\partial t^{2}}+2 \Gamma_{e} \frac{\partial n_{1}\left(\omega_{ \pm}, k_{ \pm}\right)}{\partial t}+\bar{\omega}_{p}^{2} n_{1}\left(\omega_{ \pm}, k_{ \pm}\right)-i k \frac{n_{0} e}{m} \delta_{1} \delta_{3} \delta_{4} E_{p l} \\
=-i k \bar{E} \delta_{2} n_{1}\left(\omega_{ \pm}, k_{ \pm}\right)
\end{array}
$$

where

$$
\begin{aligned}
& \delta_{1}=\frac{\omega_{0, p o l}^{2}}{\omega_{0, p o l}^{2}-\omega_{p}^{2}-\omega_{c}^{2}}, \delta_{2}=\frac{\omega_{0}^{2}}{\omega_{0}^{2}-\omega_{p}^{2}-\omega_{c}^{2}}, \\
& \delta_{3}=\frac{\omega_{q}^{2}}{\left(-\omega_{p o l}^{2}+\omega_{0, p o l}^{2}-2 i \Gamma_{p o l} \omega_{p o l}\right)}, \delta_{4}=\frac{\omega_{c}^{2}}{4 \Gamma_{p o l}^{2}-\omega_{c}^{2}}, \\
& \omega_{q}^{2}=\frac{1}{\varepsilon_{0}}\left[\frac{\left(n_{1} e q_{s}-N q_{s}^{2}\right)}{M}+\frac{\left(N e q_{s}-n_{0} e^{2}\right)}{m}\right], \\
& \bar{E}=-\left(\frac{e}{m} E_{0}+\omega_{c} v_{0 z}\right), \text { and } \bar{\omega}_{p}^{2}=\delta_{1} \omega_{p}^{2} .
\end{aligned}
$$

In deriving Eq. (9), we considered only the resonant side band frequencies $\left(\omega_{0} \pm \omega_{\text {pol }}\right)$ by assuming a long interaction path (i.e. semiconductor plasma of infinite extent), so that the higher order scattering terms, being off resonant, may be safely neglected [25], and the only waves coupled by the polaron mode are the incident $\left(\omega_{0}\right)$ and scattered wave at $\omega_{0} \pm \omega_{\text {pol }}$. Using Eq. (9), these side bands are forced waves can be expressed as

$$
n_{1}\left(\omega_{+}, k_{+}\right)=i k n_{0}(e / m) \delta_{1} \delta_{3} \delta_{4}\left(Z_{+}\right)^{-1} E_{p o l},
$$

and

$$
n_{1}\left(\omega_{-}, k_{-}\right)=i k n_{0}(e / m) \delta_{1} \delta_{3} \delta_{4}\left(Z_{-}\right)^{-1} E_{p o l} .
$$

In above Eqs., $Z_{+}=\bar{\omega}_{p}^{2}-\omega_{+}^{2}-2 i \Gamma_{e} \omega_{+}+i k \delta_{2} \bar{E}, \quad$ and $Z_{-}=\bar{\omega}_{p}^{2}-\omega_{-}^{2}-2 i \Gamma_{e} \omega_{-}+i k \delta_{2} \bar{E}$, in which $\omega_{+}=\omega_{0}+\omega_{p o l}$ and $\omega_{-}=\omega_{0}-\omega_{\text {pol }}$ represent the frequencies corresponding to upper and lower side bands, respectively. In deriving Eqs. (10a, b), we further assumed that the electron density perturbations at side band frequencies $n_{1}\left(\omega_{ \pm}, k_{ \pm}\right)$vary as $\exp \left[i\left(k_{ \pm} x-\omega_{ \pm} t\right)\right]$. Eqs. $(10 \mathrm{a}, \mathrm{b})$ reveals that the side band waves are coupled to the polaron mode via density perturbation under the influence 
of strong pump field. It is also evident from above expressions that $n_{1}\left(\omega_{ \pm}, k_{ \pm}\right)$depend upon the magnitude of the pump amplitude (via $\bar{E}$ ). The density perturbations thus produced at the side band frequencies affect the propagation characteristics of the generated waves, which can be examined by employing the general electromagnetic wave equation.

The induced nonlinear current densities for the upper and lower side bands are given by

$$
J_{+}\left(\omega_{+}\right)=-e n_{1}\left(\omega_{+}\right) v_{0}
$$

and

$$
J_{-}\left(\omega_{-}\right)=-e n_{1}\left(\omega_{-}\right) v_{0}^{*} .
$$

The time integral of the nonlinear current density $J\left(\omega_{ \pm}\right)$ yield the induced polarization $P\left(\omega_{ \pm}\right)$at the modulating frequencies as

$$
P\left(\omega_{ \pm}\right)=\int J\left(\omega_{ \pm}\right) d t .
$$

The effective nonlinear polarization of the modulated wave is obtained by

$$
P\left(\omega_{ \pm}\right)=P\left(\omega_{+}\right)+P\left(\omega_{-}\right) .
$$

Here, it should be pointing out that for the amplification of the modulated waves, both the side bands should contribute equally, and this modulation is then transferred to the polaron mode, which in turn, gets amplified. Thus from Eqs. (10) (13), we obtain the total effective polarization as

$$
P_{e}=\frac{i k e \omega_{p}^{2} \varepsilon_{0} \omega_{0} \delta_{1} \delta_{3} \delta_{4}\left(Z_{1}\right)\left|E_{0}\right|\left|E_{p o l}\right|}{m^{2}\left(\omega_{0}^{2}-\omega_{c}^{2}\right)^{2}},
$$

where

$Z_{1}=\frac{1}{\omega_{+}}\left(\Omega_{1}^{2}-2 i \Gamma_{e} \omega_{+}+i k \delta_{2} \bar{E}\right)^{-1}+\frac{1}{\omega_{-}}\left(\Omega_{2}^{2}-2 i \Gamma_{e} \omega_{-}+i k \delta_{2} \bar{E}\right)^{-1}$, in which $\Omega_{1}^{2}=\delta_{1} \omega_{p}^{2}-\omega_{+}^{2}$, and $\Omega_{2}^{2}=\delta_{1} \omega_{p}^{2}-\omega_{-}^{2}$.

Eq. (14) reveals that the total effective polarization $P_{e}$ couples to the perturbed density waves at frequencies $\omega_{ \pm}$and the polaron mode at frequency $\omega_{\text {pol }}$.

By algebraic simplification of Eq. (14), the effective thirdorder polarization $P_{e}^{(3)}$ may be expressed as

$$
\begin{aligned}
& P_{e}^{(3)}=\frac{2 k^{2}(e / m)^{2} \omega_{p}^{2} \varepsilon_{0} \delta_{1} \delta_{2} \delta_{3} \delta_{4}\left(\Omega^{2}+4 \Gamma_{e}^{2}\right)\left(Z_{2}\right)^{-1}\left|E_{0}\right|^{2}\left|E_{p o l}\right|}{\left(\omega_{0}^{2}-\omega_{c}^{2}\right)^{2}}, \\
& \quad \text { where } Z_{2}=\left(\Omega^{2}+4 \Gamma_{e}^{2}-\frac{k^{2} \delta_{2}^{2} \bar{E}^{2}}{\omega_{0}^{2}}\right)^{2}+\frac{4 k^{2} \delta_{2}^{2} \bar{E}^{2}}{\omega_{0}^{2}}, \\
& \Omega=\omega_{0}-\delta_{1} \omega_{p} .
\end{aligned}
$$

The transverse components of the oscillatory electron fluid velocity $v_{0}$ in the presence of the pump and magnetostatic fields obtained from Eq. (3) may be expressed as

$$
v_{0 x}=-\frac{\bar{E}}{2 \Gamma_{e}-i \omega_{0}} \text { and } v_{0 y}=\frac{e \omega_{c} E_{0}}{m\left(\omega_{0}^{2}-\omega_{c}^{2}\right)} .
$$

The induced third-order polarization due to cubic nonlinearities at the modulated frequencies $\left(\omega_{ \pm}\right)$may be defined as

$$
P_{e}^{(3)}=\varepsilon_{0} \chi_{e}^{(3)}\left|E_{0}\right|^{2}\left|E_{p o l}\right| \text {. }
$$

The use of Eqs. (15) in (17) yields the effective third-order optical susceptibility in the coupled mode scheme as

$$
\chi_{e}^{(3)}=\frac{2 \varepsilon_{1} k^{2}(e / m)^{2} \omega_{p}^{2} \delta_{1} \delta_{2} \delta_{3} \delta_{4}\left(Z_{2}\right)^{-1}\left(\Omega^{2}+4 \Gamma_{e}^{2}\right)}{\left(\omega_{0}^{2}-\omega_{c}^{2}\right)^{2}} .
$$

It is evident from Eq. (18) that $\chi_{e}^{(3)}$ is a complex quantity and can thus be separated into real and imaginary parts as

$\left[\chi_{e}^{(3)}\right]=\left[\chi_{e}^{(3)}\right]_{r}+i\left[\chi_{e}^{(3)}\right]_{i}$,

where

$\left[\chi_{e}^{(3)}\right]_{r}=\frac{2 \varepsilon_{1} k^{2}(e / m)^{2} \omega_{p}^{2} \omega_{q}^{2} \delta_{1} \delta_{2} \delta_{4}\left(\Omega^{2}+4 \Gamma_{e}^{2}\right)\left(\omega_{p o l}^{2}-\omega_{0, p o l}^{2}\right)\left(Z_{2}\right)^{-1}}{\left.\left[\left(\omega_{p o l}^{2}-\omega_{0, p o l}^{2}\right)^{2}+4 \Gamma_{p o l}^{2} \omega_{p o l}^{2}\right)\right]\left(\omega_{0}^{2}-\omega_{c}^{2}\right)^{2}}$,

and

$\left[\chi_{e}^{(3)}\right]_{i}=\frac{-4 \varepsilon_{1} k^{2}(e / m)^{2} \omega_{p}^{2} \omega_{q}^{2} \Gamma_{p o l} \omega_{p o l} \delta_{1} \delta_{2} \delta_{4}\left(\Omega^{2}+4 \Gamma_{e}^{2}\right)\left(Z_{2}\right)^{-1}}{\left.\left[\left(\omega_{p o l}^{2}-\omega_{0, p o l}^{2}\right)^{2}+4 \Gamma_{p o l}^{2} \omega_{p o l}^{2}\right)\right]\left(\omega_{0}^{2}-\omega_{c}^{2}\right)^{2}}$.

Here, the subscripts " $r$ " and " $i$ " to the susceptibility stand for its real and imaginary parts, respectively. The above analysis has been made for the highly doped regime, in which $\omega_{p} \approx \omega_{0}\left(\approx \omega_{ \pm}\right)$while $\omega_{p} \gg \Gamma_{e}, \omega_{0, p o l}$. Eqs. $(19 \mathrm{~b}, \mathrm{c})$ describe the steady state optical response of the medium in the presence of a transverse magnetic field and govern the nonlinear wave propagation through the medium. Now the real and imaginary parts of effective third-order susceptibility $\left(\left[\chi_{e}^{(3)}\right]_{r},\left[\chi_{e}^{(3)}\right]_{i}\right)$ can be employed to study dispersive characteristics and steady-state growth rate of the propagating modulated wave, respectively. It can be observed from Eq. (19b) that there is an intensity dependent refractive index (via $\left[\chi_{e}^{(3)}\right]_{r}$ ) leading to the possibility of a focusing or defocusing effect of the propagation beam. Eq. (19b) reveals that the positive dispersive characteristic of the dissipative medium is possible at $\omega_{p} \gg \Gamma_{e}$. As $\left[\chi_{e}^{(3)}\right]_{r}$ becomes more positive, we may expect more effective self-defocusing of the modulated polaron mode.

In order to obtain an expression for the threshold value of pump amplitude required for the onset of PL-LOP interaction based amplification of modulated waves, we set $\left[\chi_{e}^{(3)}\right]_{i}=0$ and obtain

$$
\left|E_{0, t h}\right|_{p l-l o p}=\frac{\Omega\left(\omega_{0}^{2}-\omega_{c}^{2}\right)}{(e / m) k \delta_{2} \omega_{0}} .
$$

We observed from above equation that the modulational instability of the polaron mode has a non-zero intensity threshold, even in the absence of damping. $\left(E_{0, t h}\right)_{p l-l o p}$ is found to have complex characteristics and is independent of effective charge $q_{s}$ but strongly dependent on wave number $k$, 
equilibrium carrier concentration $n_{0}$ (via $\omega_{p}$, through parameter $\Omega$ ) and the external d.c. magnetic field $B_{0}\left(\right.$ via $\left.\omega_{c}\right)$.

In order to express the possibility of PL-LOP interaction based amplification of modulated waves in a weakly-polar magnetoactive semiconductor plasmas, we employ the relation

$$
\alpha_{e}=\frac{k}{2 \varepsilon_{1}}\left[\chi_{e}^{(3)}\right]_{r}\left|E_{0}\right|^{2} .
$$

Here, $\alpha_{e}$ is the effective nonlinear absorption coefficient. The nonlinear growth of the modulated signal is possible only if $\alpha_{e}$ obtainable from Eq. (21) is negative. Thus, the growth rate of the modulated beam for pump amplitudes well above the threshold electric field can be obtained from Eqs. (19c) and (21) as

$$
\begin{aligned}
& (g)_{p l-l o p}=-\alpha_{e} \\
& \quad=\frac{2 k^{3}(e / m)^{2} \omega_{p}^{2} \omega_{q}^{2} \Gamma_{p o l} \omega_{p o l} \delta_{1} \delta_{2} \delta_{4}\left(\Omega^{2}+4 \Gamma_{e}^{2}\right)\left(Z_{2}\right)^{-1}\left|E_{0}\right|^{2}}{\left.\left[\left(\omega_{p o l}^{2}-\omega_{0, p o l}^{2}\right)^{2}+4 \Gamma_{p o l}^{2} \omega_{p o l}^{2}\right)\right]\left(\omega_{0}^{2}-\omega_{c}^{2}\right)^{2}}
\end{aligned}
$$

We observed from above equation that the growth rate $(g)_{p l}$ lop independent of its frequency $\left(\omega_{ \pm}\right)$and instead, it depends on the frequency of the pump wave $\left(\omega_{0}\right)$ as well as LOP mode $\left(\omega_{\text {lop }}\right)$. Moreover, $(g)_{\text {pl-lop }}$ is found to be influenced by the wave number $k$, equilibrium carrier concentration $n_{0}$ (via $\omega_{p}$, through parameter $\Omega$ ) and the external d.c. magnetic field $B_{0}\left(\right.$ via $\left.\omega_{c}\right)$.

\section{RESULTS AND DISCUSSION}

In order to establish the validity of present model, we have chosen a weakly-polar III-V semiconductor plasma at $77 \mathrm{~K}$ as the medium; it is assumed to be irradiated by a $10.6 \mu \mathrm{m}\left(\omega_{0}=\right.$ $1.78 \times 10^{14} \mathrm{~s}^{-1}$ ) pulsed $\mathrm{CO}_{2}$ laser. Around this temperature, absorption coefficient of the sample is low around $10 \mu \mathrm{m}$ and one may safely neglect contribution due to band-to-band transition mechanism. The physical constants involved are [26]: $m=0.014 m_{0}$, where $m_{0}$ is the free electron mass, $M=$ $2.7 \times 10^{-19} \mathrm{~kg}, N=1.48 \times 10^{28} \mathrm{~m}^{-3}, q_{s}=3.2 \times 10^{-20} \mathrm{C}, \Gamma_{e}=10^{11} \mathrm{~s}$ ${ }^{1}, \Gamma_{p h}=10^{-2} \mathrm{~s}^{-1}, \varepsilon_{1}=17.72$ and $\varepsilon_{\infty}=15.68$. This set of data is related to a typical $\mathrm{n}$-InSb crystal, however, the results obtained in previous section may be applied to any III-V semiconductor plasma.

Using the physical constants (for n-Insb) given above, the nature of dependence of the threshold pump amplitude $\left(E_{0, t h}\right)_{p l}$ lop for the onset of PL-LOP interaction based amplification of modulated waves on different parameters such as wave number $k$, externally applied magnetic field $B_{0}$ (via electron-cyclotron frequency $\omega_{c}$ ) and doping concentration $n_{0}$ (via electronplasma frequency $\omega_{p}$ ) may be studied from Eq. (20). The results are plotted in Figs. $1-3$.

Fig. 1 shows the variation of $\left(E_{0, t h}\right)_{p l-l o p}$ with wave number $k$ at $\omega_{c}, \omega_{p} \sim \omega_{0}$. It can be observed that $\left(E_{0, t h}\right)_{p l-l o p}$ is comparatively larger for lower magnitudes wave number. With increasing wave number magnitude, the threshold pump amplitude decreases parabolically. This behaviour may be attributed to the fact that $\left(E_{0, t h}\right)_{p l-l o p} \propto k^{-1}$ as suggested from Eq. (20).

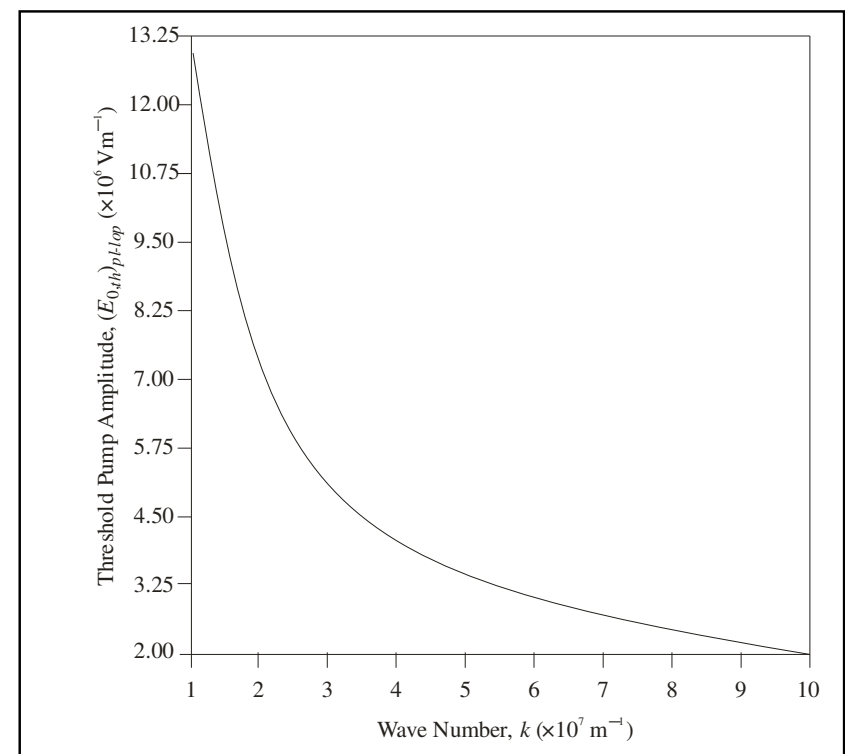

Fig. 1: Variation of $\left(E_{0, t h}\right)_{p l-l o p}$ with $k$ at $\omega_{c}, \omega_{p} \sim \omega_{0}$.

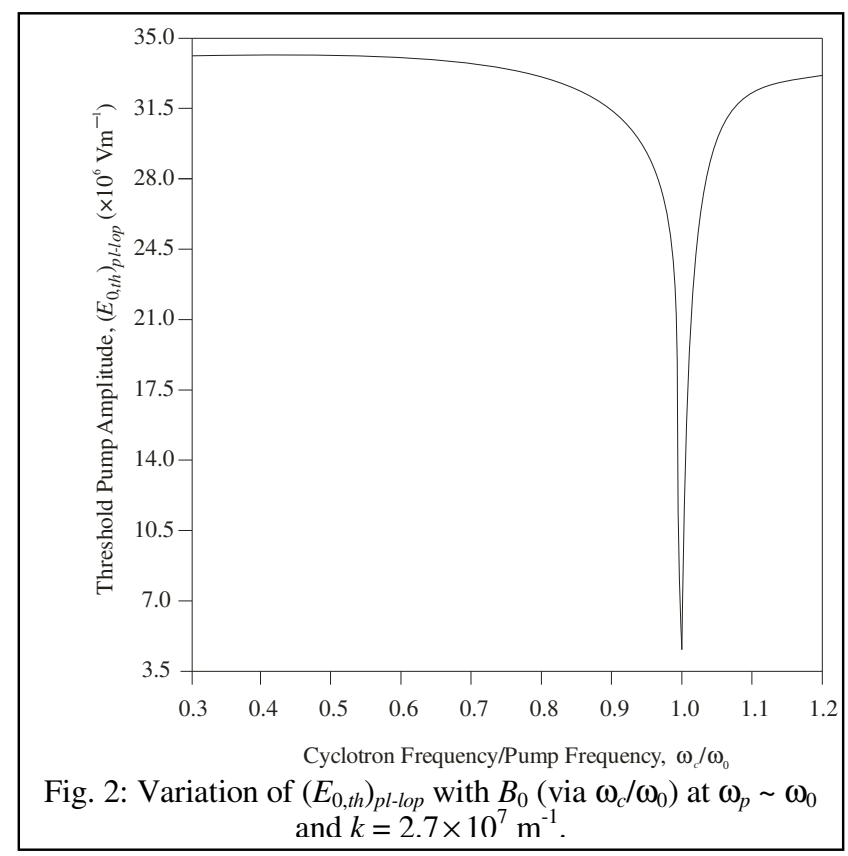

Fig. 2 shows the variation of $\left(E_{0, t h}\right)_{p l-l o p}$ with externally applied magnetic field $B_{0}\left(\right.$ via $\left.\omega_{c} / \omega_{0}\right)$ at $\omega_{p} \sim \omega_{0}$. It can be seen that $\left(E_{0, t h}\right)_{\text {pl-lop }}$ is fairly independent on magnetic field when $\omega_{c}$ $<\omega_{0}$. An increase in the value of magnetic field causes sharp fall in $\left(E_{0, t h}\right)_{p l-l o p}$. Around $\omega_{c} \sim \omega_{0},\left(E_{0, t h}\right)_{p l-l o p}$ attains a minimum value $\left(\sim 4 \times 10^{6} \mathrm{Vm}^{-1}\right.$ for $\mathrm{n}$-InSb crystal when irradiated by $\mathrm{CO}_{2}$ laser of frequency $1.78 \times 10^{14} \mathrm{~s}^{-1}$; corresponding magnetic field $\left.B_{0}=14.2 \mathrm{~T}\right)$. The occurrence of this minimum may be attributed to the fact that $\left(E_{0, t h}\right)_{p l-l o p} \propto\left(\omega_{0}^{2}-\omega_{c}^{2}\right)$ as evident from Eq. (20). With further increase in the value of magnetic field causes sharp increase in $\left(E_{0, t h}\right)_{p l-l o p}$, making it independent on magnetic field when $\omega_{c}>\omega_{0}$. 


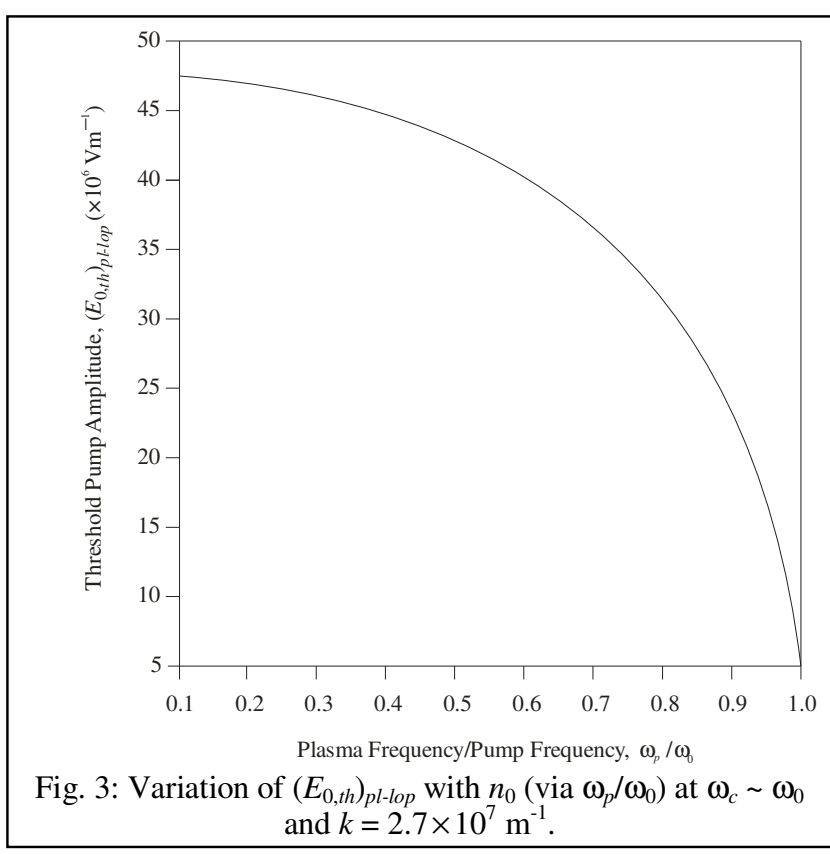

Fig. 3 shows the variation of $\left(E_{0, t h}\right)_{p l-l o p}$ with doping concentration $n_{0}\left(\right.$ via $\left.\omega_{p} / \omega_{0}\right)$ at $\omega_{c} \sim \omega_{0}$. It can be seen that $\left(E_{0, t h}\right)_{p l-l o p}$ decreases with increasing doping concentration. For $\omega_{p}<\omega_{0}$, the rate of decrease of threshold pump amplitude with respect to doping concentration is smaller; however, it increases with rise in doping concentration. Around $\omega_{p} \sim \omega_{0}$, $\left(E_{0, t h}\right)_{p l-l o p}$ attains a minimum value $\left(\sim 5 \times 10^{6} \mathrm{Vm}^{-1}\right.$ for $\mathrm{n}$-InSb crystal when irradiated by $\mathrm{CO}_{2}$ laser of frequency $1.78 \times 10^{14} \mathrm{~s}^{-}$ ${ }^{1}$; corresponding doping concentration $n_{0}=2.2 \times 10^{24} \mathrm{~m}^{-3}$ ). The occurrence of this minimum may be attributed to the fact that $\left(E_{0, t h}\right)_{p l-l o p} \propto \Omega\left[=\omega_{0}-\delta_{1} \omega_{p}\right]$ as evident from Eq. (20). The doping concentrations for which the plasma frequency $\omega_{p}$ exceeds the input pump frequency $\omega_{0}$ have been not plotted in this figure because, in the regime when $\omega_{p}>\omega_{0}$, the diffusion effects may pronounce and the theoretical formulation thereby needs a modification.

Using the material parameters (for n-Insb) given above, the nature of dependence of growth rate $(g)_{p l-l o p}$ of modulationally amplified wave on different parameters such as wave number $k$, externally applied magnetic field $B_{0}$ (via electron-cyclotron frequency $\omega_{c}$ ), doping concentration $n_{0}$ (via electron-plasma frequency $\omega_{p}$ ) and pump field amplitude $E_{0}$ (well above the threshold pump amplitude) may be studied from Eq. (22). The results are plotted in Figs. $4-7$.

Fig. 4 shows the variation of $(g)_{\text {pl-lop }}$ with wave number $k$ at $\omega_{c}, \omega_{p} \sim \omega_{0}$ and $E_{0}=2.5 \times 10^{8} \mathrm{Vm}^{-1}$. The growth rate $(g)_{p l-l o p}$ has the usual characteristic dependence on the wave vector. For lower magnitudes of wave number $k\left(\leq 6 \times 10^{7} \mathrm{~m}^{-1}\right),(g)_{p l-l o p}$ shows a linear gradual increase with $k$. With further increasing the magnitude of wave number $k$, the growth rate $(g)_{p l-l o p}$ increases quadrically. This variation of the temporal growth rate $(g)_{p l-l o p}$ with wave number $k$ is in confirmation with the usual dependence quoted above.

Fig. 5 shows the variation of $(g)_{p l-l o p}$ with externally applied magnetic field $B_{0}$ (via $\left.\omega_{c} / \omega_{0}\right)$ at $\omega_{p} \sim \omega_{0}$ and $E_{0}=$ $2.5 \times 10^{8} \mathrm{Vm}^{-1}$. It can be observed that initially $(g)_{p l-l o p}$ is very
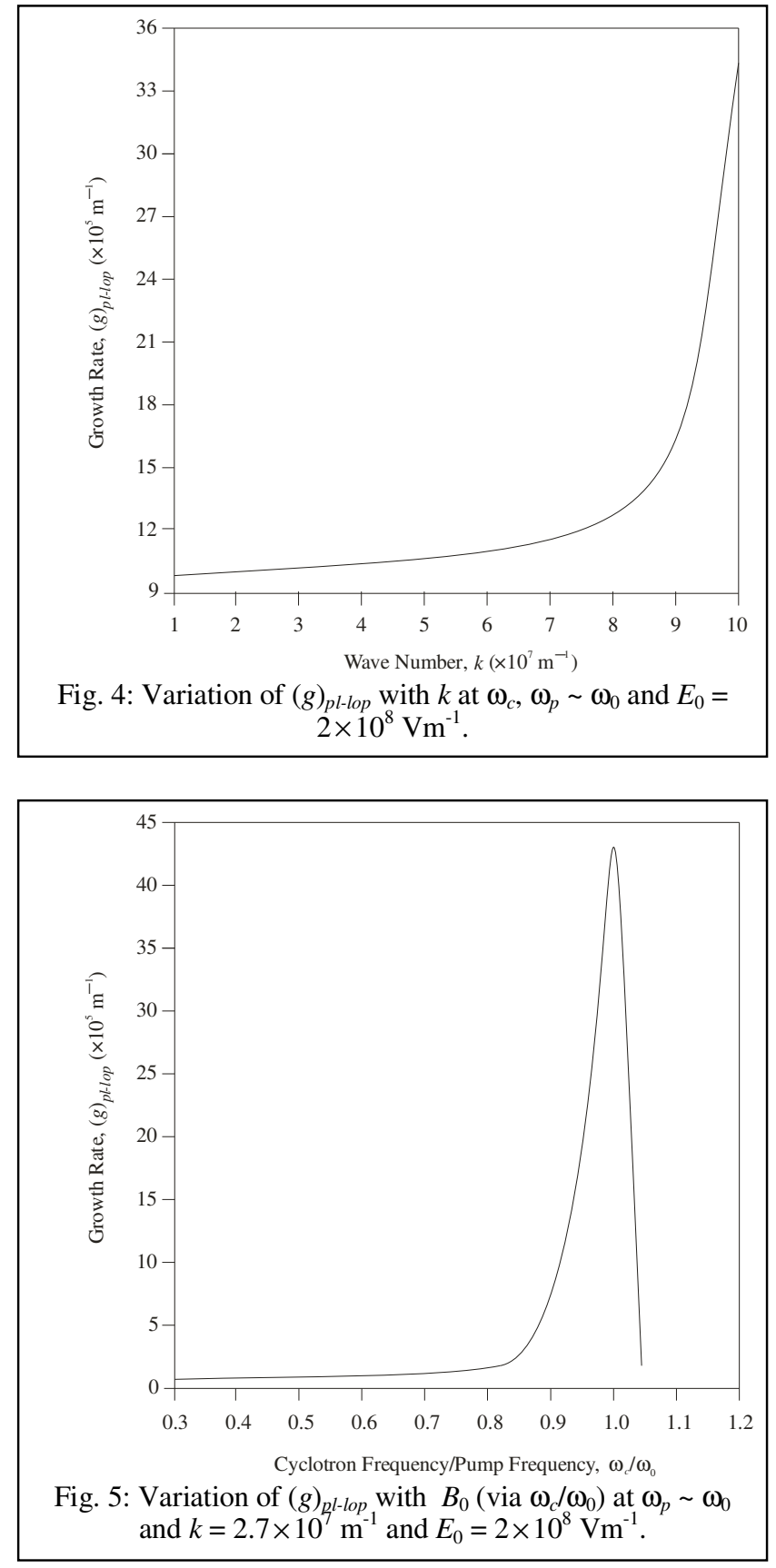

small and nearly independent of externally applied magnetic field up to $\omega_{c}=0.8 \omega_{0}$. As the magnetic field increases, $(g)_{p l-l o p}$ increases rapidly with an increase in the electron cyclotron frequency, until it reaches its maximum at $\omega_{c} \sim \omega_{0}$, and then decreases suddenly, and achieves its minimum at $\omega_{c}=1.05 \omega_{c}$. The occurrence of the peak of $(g)_{p l-l o p}$ around $\omega_{c} \sim \omega_{0}$ may be attributed to fact that $(g)_{p l-l o p} \propto\left(\omega_{0}^{2}-\omega_{c}^{2}\right)^{-2}$ as evident from Eq. (22). The sudden fall of $(g)_{p l-l o p}$ around $\omega_{c}=1.05 \omega_{0}$ may be attributed to increase in factor $\left(\omega_{0}^{2}-\omega_{c}^{2}\right)^{2}$ in the denominator of Eq. (22). Prominent amplification characteristics reveal that no growth rate could be achieved beyond $\omega_{c}=1.05 \omega_{0}$ because in this regime of magnetic field the phenomenon of cyclotron absorption may dominate the instability process and hence the 

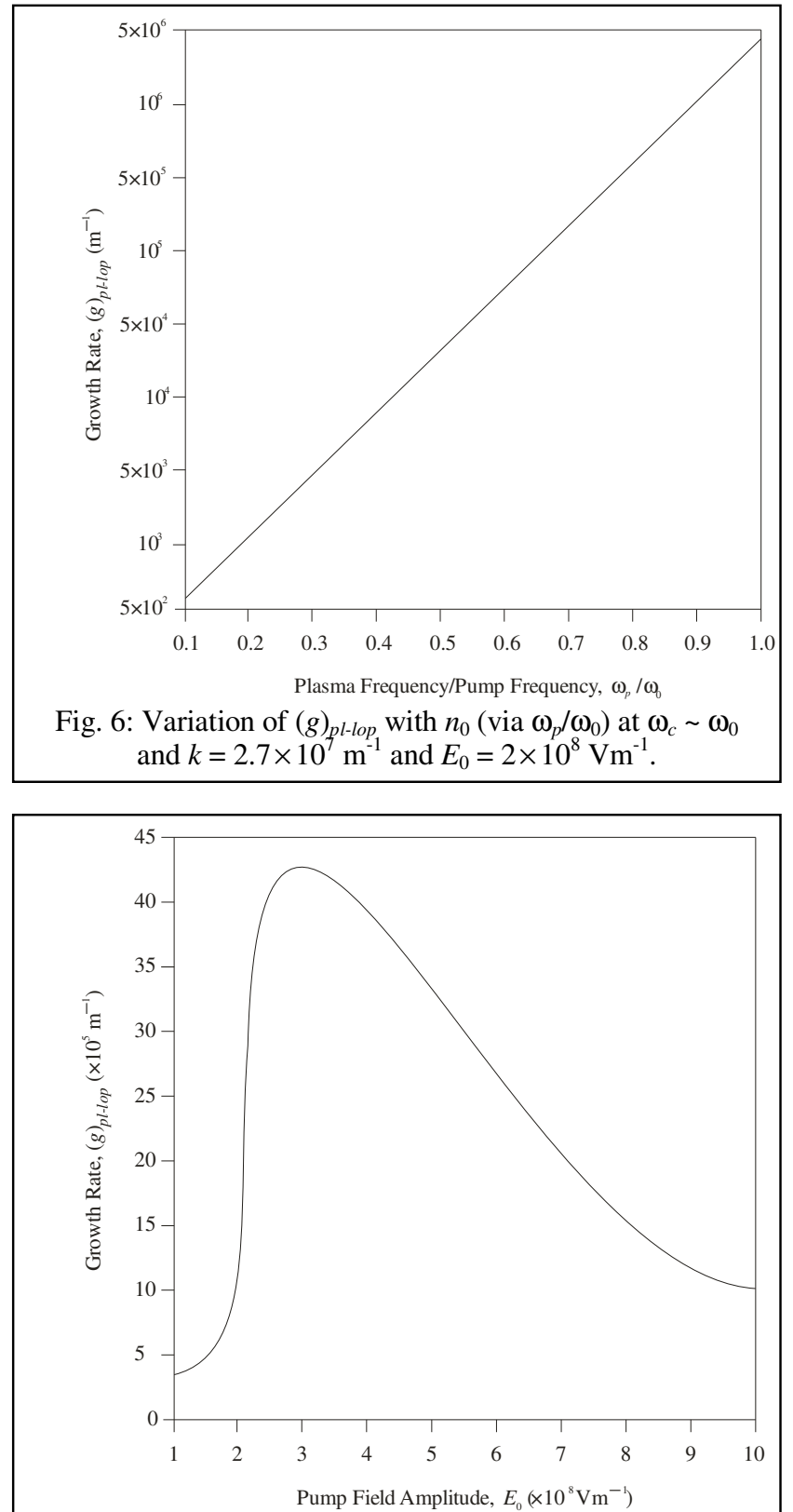

Fig. 7: Variation of $(g)_{p l-l o p}$ with $E_{0}$ at $\omega_{c}, \omega_{p} \sim \omega_{0}$ and $k=$ $2.7 \times 10^{7} \mathrm{~m}^{-1}$.

growth rate of PL-LOP interaction based modulationally amplified wave vanishes. Washing out of growth rate at and above $\omega_{c}=1.05 \omega_{0}$ provides a favourable range of magnetic field which becomes more and more confined with increasing pump field amplitudes. The above said variation of growth rate may very easily be utilized in the construction of optical switches.

Fig. 6 shows the variation of $(g)_{p l-l o p}$ with doping concentration $n_{0}\left(\right.$ via $\left.\omega_{p} / \omega_{0}\right)$ at $\omega_{c} \sim \omega_{0}$ and $E_{0}=2.5 \times 10^{8} \mathrm{Vm}^{-1}$ $\mathrm{Vm}^{-1}$. It can be observed that $(g)_{p l-l o p}$ is very small $\left(\sim 10^{2} \mathrm{~m}^{-1}\right)$ for low doping concentrations $\left(\omega_{p} \sim 0.1 \omega_{c}\right.$, corresponding $n_{0}=$ $\left.2.2 \times 10^{22} \mathrm{~m}^{-3}\right)$. It increases linearly with rise in electron density of the semiconductor plasma. At high doping concentrations $\left(\omega_{p} \sim \omega_{0}\right.$, corresponding $\left.n_{0}=2.2 \times 10^{24} \mathrm{~m}^{-3}\right),(g)_{p l-l o p}$ is very high $\left(\sim 10^{6} \mathrm{~m}^{-1}\right)$. Hence, $(g)_{p l-l o p}$ strongly depends on electron density of the semiconductor plasma. The nature of the $(g)_{p l-l o p}$ - $\omega_{p}$ graph is similar to conclusions arrived at by Salimullah and Singh [27] who considered the interaction of an extraordinary mode subjected to perturbations parallel to the magnetic field. Thus higher growth rate of modulationally amplified wave can be attained by increasing the doping concentration of the medium by n-type doping in the crystal. However the doping should not exceed the limit for which the plasma frequency $\omega_{p}$ exceeds the input pump frequency $\omega_{0}$, because, in the regime when $\omega_{p}>\omega_{0}$, the electromagnetic pump wave will be reflected back by the intervening medium. It may be thereby concluded that semiconductor plasmas are the most appropriate hosts for modulational instability processes.

The preceding analysis has been performed for III-V semiconductor plasmas like $\mathrm{n}$-InSb with doping concentrations approaching critical density (i.e. doping concentrations for which the corresponding electron-plasma frequency is comparable to the incident pump frequency $\omega_{p} \sim \omega_{0}$ ). Doping concentrations of such high magnitudes are quite relevant to semiconductors of the III-V group [28] and have been extensively employed by several workers to study their various characteristics [29].

Fig. 7 shows the variation of $(g)_{p l-l o p}$ with pump field amplitude $E_{0}$ at $\omega_{c}, \omega_{p} \sim \omega_{0}$. It can be observed from this figure that $(g)_{p l-l o p}$ increases sharply with $E_{0}$, achieving a peak at $E_{0}=$ $3 \times 10^{8} \mathrm{Vm}^{-1}$. Beyond this value of pump field amplitude, $(g)_{p l}$ lop decreases parabolically with pump field amplitude. Thus, by suitably choosing the pump field amplitude, maximum growth rate of PL-LOP interaction based modulationally amplified wave can be obtained in a semiconductor plasma.

\section{CONCLUSIONS}

In the present study, a detailed numerical analysis of the threshold pump field for the onset of PL-LOP interaction based amplification of modulated waves and growth rate of modulated wave well above the threshold pump amplitude in magnetoactive semiconductor plasmas have been undertaken. The analysis enables us to draw following conclusions:

1. The hydrodynamic model of semiconductor-plasma has been successfully applied to study the influence of different parameters such as externally applied magnetostatic field, doping concentration etc. on threshold pump amplitude and growth rate of modulated wave (well above the threshold pump amplitude) in magnetoactive semiconductor plasmas duly shined by slightly off-resonant not too high power pulsed lasers.

2. The threshold pump amplitude for the onset of PLLOP interaction based amplification of modulated waves decreases with increasing the wave number amplitudes. The presence of an external transverse d.c. magnetic field (for which electron cyclotron frequency $\sim$ pump wave frequency) and high values of doping concentrations (for which electron plasma frequency $\sim$ pump wave frequency) effectively reduces the threshold pump amplitude required for inciting the amplification of modulated waves. 
3. The growth rate of modulationally amplified wave is found to increase with wave number of LOP mode. The presence of an external transverse d.c. magnetic field (for which electron cyclotron frequency pump wave frequency) effectively enhances the growth rate of modulationally amplified wave. High levels of doping concentration are favourable to large growth rate.

4. The increment in pump field amplitude increases the growth rate of PL-LOP interaction based modulationally amplified wave, resulting in peak at $E_{0}$ $=3 \times 10^{8} \mathrm{Vm}^{-1}$.

\section{ACKNOWLEDGMENT}

The authors are thankful to Dr. Manjeet Singh, Department of Physics, Govt. P.G. Nehru College, Jhajjar (Haryana) for his kind cooperation.

\section{REFERENCES}

[1] D.S. Chemla, D.A.B. Miller, S. Schmitt-Rink, Optical Nonlinearities and Instabilities in Semiconductors. New York, Academic Press, (1988).

[2] T. Meier, P. Thomas, S.W. Koch, Coherent Semiconductor Optics: From Basic Concepts to Nanostructure Applications. New York, Springer, (2007).

[3] P. Yu, M. Cardona, Fundamentals of Semiconductor - Physics and Materials Properties. Berlin, Springer, (1996).

[4] G.P. Agarwal, Nonlinear Fiber Optics. Boston, Academic Press, (1984).

[5] E. Garmire, "Resonant optical nonlinearities in semiconductors," IEEE Journal of Selected Topics in Quantum Electron. vol. 6, pp. 1094-1110, 2000 .

[6] A. Mooradian, G.B. Wright, "Observation of the interaction of plasmons with longitudinal optical phonons in GaAs," Phys. Rev. Lett. vol 16, pp. 999-1001, 1966.

[7] R. Kaplan, E.D. Palik, R.F. Wallis, S. Iwasa, E. Burstein, "Infrared absorption by coupled collective cyclotron-longitudinal-optic phonon modes in InSb," Phys. Rev. Lett. vol. 18, pp. 159-162, 1967.

[8] J.T. Devreese, Polaron, in Encyclopedia of Physics. R. Lerner, G. Trigg (eds.). Boston, Addison-Wesley Publishing Company (1981).

[9] X.Q. Zhu, J.J. Shi, Z.X. Liu, S.H. Pan, "Energy and effective mass of a polaron in asymmetric semiconductor quantum well structures," Z. Phys. B vol. 102, pp. 207-216, 1997.

[10] J. Heyman, P.A. Crowell, T. Mueller, K. Unterrainer, "Terahertz emission from GaAs and InAs in a magnetic field," Phys. Rev. B vol. 64, pp. 085202 (7pp), 2001

[11] M.B. Johnston, A. Corchia, A. Dowd, E.H. Linfield, "Magnetic-fieldinduced enhancement of terahertz emission from III-V semiconductor surfaces," Physica E: Low-dim. Syst. Nanostruct. vol. 13, pp. 896-899, 2002.

[12] I. Nevinskas, F. Kadlec, C. Kadlec, R. Butkute, A. Krotkus, "Terahertz pulse emission from epitaxial n-InAs in a magnetic field," J. Phys. D: Appl. Phys. vol. 52, pp. 365301 (5pp), 2019.

[13] R. Kaplan, E.D. Palik, R.F. Wallis, S. Iwasa, E. Burstein, "Infrared absorption by coupled collective cyclotron excitation-longitudinal-optic phonon modes in InSb," Phys. Rev. Lett. vol. 18, pp. 159-162, 1967.

[14] A. Neogi, "Acousto-optic modulation in diffusive semiconductors," J. Appl. Phys. vol. 77, pp. 327-333, 1994.

[15] S. Ghosh, M.P. Rishi, "Acousto-optic modulation in magnetized diffusive semiconductors," Eur. Phys. J. D vol 19, pp. 223-230, 2002

[16] S. Ghosh, M.P. Rishi, "Acousto-optic amplitude modulation and demodulation of electromagnetic wave in magnetized diffusive semiconductor plasma," Eur. Phys. J D vol. 20, pp. 275-279, 2002.

[17] N. Nimje, S. Dubey, S.K. Ghosh, "Diffusion-induced modulational instability in magnetized semiconductor plasmas: effects of carrier heating,” Eur. Phys. J. D vol. 59, pp. 223-231, 2010.
[18] M. Hase, M. Katsuragawa, A.M. Constantinescu, H. Petek, "Coherent phonon-induced optical modulation in semiconductors at terahertz frequencies,” New J. Phys. vol. 15, pp. 055018 (16pp), 2013.

[19] F. Likawa, A.H. Minguez, M. Ramsteiner, P.V. Santos, "Optical phonon modulation in semiconductors by surface acoustic waves," Phys. Rev. B vol. 93, pp. 195212 (7pp), 2016.

[20] P.K. Chakraborty, B.N. Mondal, B.K. Chaudrari, "Laser-induced modulation of optical band-gap parameters in the III-V type semiconductors from the density-of-states (DOS) calculations," Pramana- J. Phys. vol. 92, pp. 85 (11pp), 2019.

[21] P.K. Kaw, "Parametric excitation of ultrasonic waves in piezoelectric semiconductors," J. Appl. Phys. vol. 14, pp. 1497-1498, 1973.

[22] A.V. Kuznetsov, C.J. Stanton, "Coherent phonon oscillations in GaAs," Phys. Rev. B vol. 51, pp. 7555-7565, 1995.

[23] K. Seeger, Semiconductor Physics. Berlin, Springer, (1989).

[24] M. Singh, D. Joseph, S. Duhan, "Nonlinear optical parameters of magnetoactive semiconductor-plasmas," Int. J. Mod. Phys. B vol. 22, pp. 3877-3887, 2008.

[25] A. Yariv, Optical Electronics. New York, Holt-Saunders International, (1985).

[26] S. Jangra, H.P. Singh, V. Kumar, "Acoustical phonon and polaron-mode induced optical parametric parametric amplification in transversely magnetized III-V semiconductors," Mod. Phys. Lett. B vol. 33, pp. 1950271 (16pp), 2019.

[27] M. Salimullah, T. Singh, "Filamentation and modulational instabilities of laser radiation in magnetoactive piezoelectric semiconductors," J. Phys. Chem. Solids vol. 43, pp. 1087-1092, 1982.

[28] P.K. Sen, N. Apte, S. Guha, "Raman instability in n-type piezoelectric semiconductors," Phys. Rev. vol. 22, pp. 6340-6346, 1980.

[29] M. Singh, P. Aghamkar, S. Duhan, "Enhancement of second- and thirdorder nonlinear optical susceptibilities in magnetized semiconductors," Chin. Phys. Lett. vol. 25, pp. 3276-3279, 2008. 